\title{
Word Automaticity of Tree Automatic Scattered Linear Orderings Is Decidable
}

\author{
Martin Huschenbett \\ Institut für Theoretische Informatik, Technische Universität Ilmenau, Germany \\ martin.huschenbett@tu-ilmenau.de
}

\begin{abstract}
A tree automatic structure is a structure whose domain can be encoded by a regular tree language such that each relation is recognisable by a finite automaton processing tuples of trees synchronously. Words can be regarded as specific simple trees and a structure is word automatic if it is encodable using only these trees. The question naturally arises whether a given tree automatic structure is already word automatic. We prove that this problem is decidable for tree automatic scattered linear orderings. Moreover, we show that in case of a positive answer a word automatic presentation is computable from the tree automatic presentation.
\end{abstract}

\section{Introduction}

The fundamental idea of automatic structures can be traced back to the 1960s when Büchi, Elgot, Rabin, and others used finite automata to provide decision procedures for the first-order theory of Presburger arithmetic $(\mathbb{N} ;+)$ and several other logical problems. Hodgson generalised this idea to the concept of automaton decidable first-order theories. Independently of Hodgson and inspired by the successful employment of finite automata and their methods in group theory, Khoussainov and Nerode [4 initiated the systematic investigation of automatic structures. Recalling the efforts from the 1960s, Blumensath [2] extended this concept notion beyond finite automata to finite automaton models recognising infinite words, finite trees, or infinite trees.

Basically, a countable relational structure is tree automatic or tree automatically presentable if its elements can be encoded by finite trees in such a way that its domain and its relations are recognisable by finite automata processing either single trees or tuples of trees synchronously. A structure is word automatic if its elements can be encoded using only specific simple trees which effectively represent words. In contrast to the more general concept of computable structures and based on the strong closure properties of recognisability, automatic structures provide pleasant algorithmic features. In particular, they possess decidable first-order theories.

Due to this latter fact, the concept of automatic structures gained a lot attention which led to noticeable progress (cf. [16]). Automatic presentations were found for many structures, some structures where shown to be tree but not word 
automatic, for instance Skolem arithmetic $(\mathbb{N} ; \times)$, whereas other structures, like the random graph, were proven to be neither word nor tree automatic. For some classes of structures it was even possible to characterise its automatic members, for example an ordinal is word automatic respectively tree automatic precisely if it is less than $\omega^{\omega}$ respectively $\omega^{\omega^{\omega}}$. Certain extensions of first-order logic were shown to preserve decidability of the corresponding theory. The question whether two automatic structures are isomorphic turned out to be highly undecidable in general as well as for some restricted classes of structures. At the same time, the isomorphism problem for word automatic ordinals was proven to be decidable. Last but not least, the different classes of automatic structures was characterised by means of interpretations in universal structures.

Due to the fact that word automaticity is a special case of tree automaticity, the question naturally arises whether a given tree automatic structure is already word automatic. As far as we know, this problem was neither solved in general nor for any restricted class of structures. For that reason, we investigate the respective question for scattered linear orderings in this paper. Actually, we prove the corresponding problem to be decidable and our main result is as follows:

Theorem 1.1. Given a tree automatic presentation $\mathcal{P}$ of a scattered linear ordering $\mathfrak{L}$, it is decidable whether $\mathfrak{L}$ is word automatic. In case $\mathfrak{L}$ is word automatic, one can compute a word automatic presentation of $\mathfrak{L}$ from $\mathcal{P}$.

Since every well-ordering is scattered, this result still holds if $\mathfrak{L}$ is assumed to be an ordinal. The proof of Theorem 1.1 splits into three parts. First, we introduce the notion of slim tree languages and prove this property to be decidable (Theorem 3.2). Second, we show that a slim domain is sufficient for a tree automatic structure to be word automatic (Theorem 4.1). Last, we demonstrate that this condition is also necessary in case of scattered linear orderings (Theorem [5.1). Altogether, Theorem 1.1 follows from the three mentioned theorems 1

\section{Background}

In this section we recall the necessary notions of logic, automatic structures (cf. [16]), tree automata (cf. [3]), and linear orderings. We agree that the natural numbers $\mathbb{N}$ include 0 and that $[m, n]=\{m, m+1, \ldots, n\} \subseteq \mathbb{N}$ for all $m, n \in \mathbb{N}$.

Logic. A (relational) signature $\tau=(\mathcal{R}$, ar) is a finite set $\mathcal{R}$ of relation symbols together with a map ar: $\mathcal{R} \rightarrow \mathbb{N}$ assigning to each $R \in \mathcal{R}$ its arity $\operatorname{ar}(R) \geq 1$. A $\tau$-structure $\mathfrak{A}=\left(A ;\left(R^{\mathfrak{A}}\right)_{R \in \mathcal{R}}\right)$ consists of a set $A=\operatorname{dom}(\mathfrak{A})$, its domain, and an $\operatorname{ar}(R)$-ary relation $R^{\mathfrak{A}} \subseteq A^{\operatorname{ar}(R)}$ for each $R \in \mathcal{R}$. First order logic $\mathrm{FO}_{\tau}$ over $\tau$ is defined as usual, including an equality predicate. A sentence is a formula without free variables. Writing $\varphi(\bar{x})$ means that all free variables of the formula $\varphi$ are among the entries of the tuple $\bar{x}=\left(x_{1}, \ldots, x_{n}\right)$. The set $\varphi^{\mathfrak{A}}$ is comprised of all $\bar{a} \in A^{n}$ satisfying $\mathfrak{A} \models \varphi(\bar{a})$, where the latter is defined as usual.

\footnotetext{
${ }^{1}$ Proofs of all seemingly unproven lemmas as well as the interpretations from Section 4 can be found in the appendix.
} 
Automatic Structures. The set of all (finite) words over an alphabet $\Sigma$ is $\Sigma^{\star}$, the empty word is $\varepsilon$, and the length of $w$ is $|w|$. Subsets of $\Sigma^{\star}$ are called languages and $L \subseteq \Sigma^{\star}$ is regular if it can be recognised by some (non-deterministic) finite automaton.

Let $\square \notin \Sigma$ be a new symbol and $\Sigma_{\square}=\Sigma \cup\{\square\}$. For $n \geq 1$ consider an $n$-tuple $\bar{w}=\left(w_{1}, \ldots, w_{n}\right) \in\left(\Sigma^{\star}\right)^{n}$ of words with $w_{i}=a_{i, 1} a_{i, 2} \ldots a_{i, m_{i}}$ for all $i \in[1, n]$. Let $m=\max \left\{m_{1}, \ldots, m_{n}\right\}$ and $a_{i, j}=\square$ for $j \in\left[m_{i}+1, m\right]$. The convolution of $\bar{w}$ is the word $\otimes \bar{w}=\bar{a}_{1} \ldots \bar{a}_{m} \in\left(\Sigma_{\square}^{n}\right)^{\star}$ with $\bar{a}_{j}=\left(a_{1, j}, \ldots, a_{n, j}\right) \in \Sigma_{\square}^{n}$ for all $j \in[1, m]$. An $n$-ary relation $R \subseteq\left(\Sigma^{\star}\right)^{n}$ is automatic if the language $\otimes R \subseteq\left(\Sigma_{\square}^{n}\right)^{\star}$, which is comprised of all $\otimes \bar{w}$ with $\bar{w} \in R$, is regular.

A $\tau$-structure $\mathfrak{A}$ with $\operatorname{dom}(\mathfrak{A}) \subseteq \Sigma^{\star}$ is (word) automatic if $\operatorname{dom}(\mathfrak{A})$ is regular and $R^{\mathfrak{A}}$ is automatic for all $R \in \mathcal{R}$. A (word) automatic presentation of $\mathfrak{A}$ is a tuple $\left(\mathcal{A}_{\text {dom }} ;\left(\mathcal{A}_{R}\right)_{R \in \mathcal{R}}\right)$ of finite automata such that $\mathcal{A}_{\text {dom }}$ recognises $\operatorname{dom}(\mathfrak{A})$ and $\mathcal{A}_{R}$ recognises $\otimes R^{\mathfrak{A}}$. Abusing notation, we call any structure $\mathfrak{B}$ which is isomorphic to some word automatic structure $\mathfrak{A}$ also (word) automatic.

Tree Automata. A tree domain is a non-empty, finite, and prefix-closed subset $D \subseteq\{0,1\}^{\star}$ satisfying $u 0 \in D$ iff $u 1 \in D$ for all $u \in D$. A tree over $\Sigma$ is a map $t: D \rightarrow \Sigma$ where $\operatorname{dom}(t)=D$ is a tree domain. The set of all trees is denoted by $T_{\Sigma}$ and its subsets are called (tree) languages. For some $t \in T_{\Sigma}$ and $u \in \operatorname{dom}(t)$ the subtree of $t$ rooted at $u$ is the tree $t\left\lceil u \in T_{\Sigma}\right.$ defined by

$$
\operatorname{dom}(t \mid u)=\left\{v \in\{0,1\}^{\star} \mid u v \in \operatorname{dom}(t)\right\} \quad \text { and } \quad(t\lceil u)(v)=t(u v) .
$$

A (deterministic bottom-up) tree automaton $\mathcal{A}=(Q, \iota, \delta, F)$ over $\Sigma$ consists of a finite set $Q$ of states, a start state function $\iota: \Sigma \rightarrow Q$, a transition function $\delta: \Sigma \times Q \times Q \rightarrow Q$, and a set $F \subseteq Q$ of accepting states. For each $t \in T_{\Sigma}$ a state $\mathcal{A}(t) \in Q$ is defined recursively by $\mathcal{A}(t)=\iota(t(\varepsilon))$ if $\operatorname{dom}(t)=\{\varepsilon\}$ and $\mathcal{A}(t)=\delta(t(\varepsilon), \mathcal{A}(t\lceil 0), \mathcal{A}(t\lceil 1))$ otherwise. The language recognised by $\mathcal{A}$ is the set of all $t \in T_{\Sigma}$ with $\mathcal{A}(t) \in F$. A language $L \subseteq T_{\Sigma}$ is regular if it can be recognised by some tree automaton.

The convolution of $\bar{t}=\left(t_{1}, \ldots, t_{n}\right) \in\left(T_{\Sigma}\right)^{n}$ is the tree $\otimes \bar{t} \in T_{\Sigma_{n}^{n}}$ defined by $\operatorname{dom}(\otimes \bar{t})=\operatorname{dom}\left(t_{1}\right) \cup \cdots \cup \operatorname{dom}\left(t_{n}\right)$ and $(\otimes \bar{t})(u)=\left(t_{1}^{\prime}(u), \ldots, t_{n}^{\prime}(u)\right)$, where $t_{i}^{\prime}(u)=t_{i}(u)$ if $u \in \operatorname{dom}\left(t_{i}\right)$ and $t_{i}^{\prime}(u)=\square$ otherwise. A relation $R \subseteq\left(T_{\Sigma}\right)^{n}$ is automatic if the language $\otimes R \subseteq T_{\Sigma_{n}^{n}}$ is regular.

Tree automatic structures and tree automatic presentations are defined like in the word automatic case, but based on trees and tree automata.

Linear Orderings. A linear ordering is a structure $\mathfrak{A}=\left(A ;<^{\mathfrak{A}}\right)$ where $<^{\mathfrak{A}}$ is a strict linear order relation on $A$. The ordering $\mathfrak{A}$ is scattered if $(\mathbb{Q} ;<)$ cannot be embedded into $\mathfrak{A}$. Obviously, every well-ordering is scattered. For any two linear orderings $\mathfrak{A}$ and $\mathfrak{B}$ we define another linear ordering $\mathfrak{A} \cdot \mathfrak{B}$ by $\operatorname{dom}(\mathfrak{A} \cdot \mathfrak{B})=$ $\operatorname{dom}(\mathfrak{A}) \times \operatorname{dom}(\mathfrak{B})$ and $\left(a_{1}, b_{1}\right)<^{\mathfrak{A} \cdot \mathfrak{B}}\left(a_{2}, b_{2}\right)$ iff either $a_{1}<^{\mathfrak{A}} a_{2}$ or $a_{1}=a_{2}$ and $b_{1}<{ }^{\mathfrak{B}} b_{2}$. Finally, if $\mathfrak{A}_{1}$ can be embedded into $\mathfrak{B}_{1}$ and $\mathfrak{A}_{2}$ into $\mathfrak{B}_{2}$, then $\mathfrak{A}_{1} \cdot \mathfrak{A}_{2}$ can be embedded into $\mathfrak{B}_{1} \cdot \mathfrak{B}_{2}$. 


\section{$3 \quad$ Slim and Fat Tree Languages}

In this section, we introduce the notion of slim tree languages and show that it is decidable whether the language recognised by a given tree automaton is slim.

Definition 3.1. The thickness $\varnothing(t)$ of a tree $t \in T_{\Sigma}$ is the maximal number of nodes on any level, i.e.,

$$
\varnothing(t)=\max \left\{\left|\operatorname{dom}(t) \cap\{0,1\}^{\ell}\right| \mid \ell \geq 0\right\} \in \mathbb{N} .
$$

For every $K \geq 1$ the set of all $t \in T_{\Sigma}$ with $\varnothing(t) \leq K$ is denoted by $T_{\Sigma, K}$. A tree language $L \subseteq T_{\Sigma}$ is slim if there exists some $K \geq 1$ such that $L \subseteq T_{\Sigma, K}$, otherwise $L$ is fat.

A tree automaton $\mathcal{A}$ is reduced if for every state $q$ of $\mathcal{A}$ there is a tree $t \in T_{\Sigma}$ with $\mathcal{A}(t)=q$. For every tree automaton $\mathcal{A}$ one can compute a reduced tree automaton which recognises the same language and has no more states than $\mathcal{A}$.

Theorem 3.2. Given a reduced tree automaton $\mathcal{A}$, it is decidable whether the tree language $L$ recognised by $\mathcal{A}$ is slim or fat. If $L$ is slim, then $L \subseteq T_{\Sigma, 2^{n-1}}$, where $n$ is the number of states of $\mathcal{A}$.

For the rest of this section we fix a reduced tree automaton $\mathcal{A}=(Q, \iota, \delta, F)$. The proof of Theorem 3.2 essentially depends on an inspection of the directed graph $G_{\mathcal{A}}=\left(Q, E_{\mathcal{A}}\right)$ with

$$
(p, q) \in E_{\mathcal{A}} \quad \text { iff } \quad \exists a \in \Sigma, r \in Q: \delta(a, p, r)=q \text { or } \delta(a, r, p)=q .
$$

Clearly, this graph is computable from $\mathcal{A}$. The lemma below is shown by applying the idea of pumping to tree automata. Therein, the height $h(t)$ of a tree $t \in T_{\Sigma}$ is the number

$$
h(t)=\max \{|u| \mid u \in \operatorname{dom}(t)\} \in \mathbb{N} .
$$

Lemma 3.3. For every $q \in Q$ the following are equivalent:

(1) there are infinitely many $t \in T_{\Sigma}$ satisfying $\mathcal{A}(t)=q$,

(2) there is a tree $t \in T_{\Sigma}$ satisfying $h(t) \geq n$ and $\mathcal{A}(t)=q$, where $n=|Q|$,

(3) $G_{\mathcal{A}}$ contains a cycle from which $q$ is reachable.

An edge $(p, q) \in E_{\mathcal{A}}$ is special if in the definition of $E_{\mathcal{A}}$ in Eq. (1) the state $r \in Q$ can be chosen such that it satisfies the conditions of Lemma 3.3 (for $r$ in place of $q$ ). Since condition (3) is decidable, it is decidable whether an edge is special. The key idea for proving Theorem 3.2 is stated by the following lemma:

Lemma 3.4. The following are equivalent:

(1) the tree language $L$ recognised by $\mathcal{A}$ is fat,

(2) there is a tree $t \in L$ satisfying $\varnothing(t)>2^{n-1}$, where $n=|Q|$,

(3) $G_{\mathcal{A}}$ contains a cycle including a special edge and from which $F$ is reachable.

The proof of this lemma works similar to the one of Lemma 3.3. Since condition (3) is decidable given $\mathcal{A}$ as input, Theorem 3.2 follows. 


\section{Slim Tree Automatic Structures Are Word Automatic}

This section is devoted to the proof of the following theorem:

Theorem 4.1. Let $\mathfrak{A}$ be a tree automatic structure such that $\operatorname{dom}(\mathfrak{A})$ is slim. Then, $\mathfrak{A}$ is already word automatic and one can compute a word automatic presentation of $\mathfrak{A}$ from a tree automatic presentation of $\mathfrak{A}$.

The idea of the proof is the following. Let $K \geq 1$ be such that $\operatorname{dom}(\mathfrak{A}) \subseteq T_{\Sigma, K}$. We give an alphabet $\widehat{\Sigma}$ and a one-to-one map $C: T_{\Sigma, K} \rightarrow \widehat{\Sigma}^{\star}$, the encoding, such that $C(L)$ is regular for all regular $L \subseteq T_{\Sigma, K}$ (Proposition 4.4) and $C(R)$ is automatic for all automatic relations $R \subseteq\left(T_{\Sigma, K}\right)^{n}$ (Proposition 4.6). Thus, the structure $C(\mathfrak{A})$ is word automatic. A word automatic presentation of $C(\mathfrak{A})$ is computable since both propositions are effective and Theorem 3.2 allows for computing a suitable $K$. Although it is possible to show both propositions using automata, it is much more convenient to accomplish this by means of logic.

\subsection{Monadic Second Order Logic}

Monadic second order logic $\mathrm{MSO}_{\tau}$ extends $\mathrm{FO}_{\tau}$ by set variables, which range over subsets of the domain and are denoted by capital letters, quantifiers for these variables, and the formula " $x \in X$ " (cf. [7]). Let $\tau=\left(\mathcal{R}\right.$, ar) and $\tau^{\prime}$ be two signatures. An (MSO-)interpretation of a $\tau$-structure $\mathfrak{A}$ in a $\tau^{\prime}$-structure $\mathfrak{B}$ is a pair $\langle f, \mathcal{I}\rangle$ comprised of a one-to-one map $f: \operatorname{dom}(\mathfrak{A}) \rightarrow \operatorname{dom}(\mathfrak{B})$ and a tuple $\mathcal{I}=\left(\Delta ;\left(\Phi_{R}\right)_{R \in \mathcal{R}}\right)$ of $\mathrm{MSO}_{\tau^{\prime}}$-formulae with free FO-variables only such that $f(\operatorname{dom}(\mathfrak{A}))=\Delta^{\mathfrak{B}}$ and $f\left(R^{\mathfrak{A}}\right)=\Phi_{R}^{\mathfrak{B}}$ for each $R \in \mathcal{R}$. In fact, $f$ induces an isomorphism between $\mathfrak{A}$ and $\mathcal{I}(\mathfrak{B})=\left(\Delta^{\mathfrak{B}} ;\left(\Phi_{R}^{\mathfrak{B}}\right)_{R \in \mathcal{R}}\right)$. Replacing in an $\mathrm{MSO}_{\tau^{-}}$ formula $\varphi(\bar{x})$ all symbols $R \in \mathcal{R}$ with $\Phi_{R}$ and relativising quantifiers to $\Delta$ yields an $\mathrm{MSO}_{\tau^{\prime}}$ formula $\varphi^{\mathcal{I}}(\bar{x})$ satisfying $\mathfrak{A} \models \varphi(\bar{a})$ iff $\mathfrak{B} \models \varphi^{\mathcal{I}}(f(\bar{a}))$ for all $\bar{a} \in A^{n}$.

For an alphabet $\Sigma$ the signature $\mathrm{W} \Sigma$ consists of one binary relation symbol $\leq$ and a unary symbol $P_{a}$ for each $a \in \Sigma$. Every word $w=a_{1} a_{2} \ldots a_{|w|} \in \Sigma^{\star}$ is regarded as a $W \Sigma$-structure with domain $\operatorname{dom}(w)=\{1, \ldots,|w|\}, \leq^{w}$ being the natural order on $\operatorname{dom}(w)$, and $i \in P_{a}^{w}$ iff $a_{i}=a$. For fixed numbers $m, r \in \mathbb{N}$, relations like $x=y+m$ and $x \equiv r(\bmod m)$ are expressible in $\mathrm{MSO}_{\mathrm{W} \Sigma}$. The

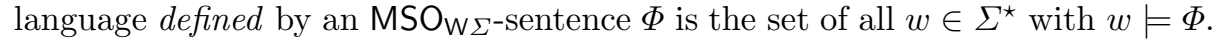

The signature $\mathrm{T} \Sigma$ is similar to $\mathrm{W} \Sigma$ but contains two binary symbols $S_{0}$ and $S_{1}$ instead of $\leq$. Each tree $t \in T_{\Sigma}$ is considered as a $T \Sigma$-structure with domain $\operatorname{dom}(t),(u, v) \in S_{d}^{t}$ iff $u d=v(d=0,1)$, and $u \in P_{a}^{t}$ iff $t(u)=a$. The language defined by some $\mathrm{MSO}_{\mathrm{T} \Sigma}$-sentence $\Phi$ is the set of all $t \in T_{\Sigma}$ with $t \models \Phi$.

The following theorem holds for word languages as well as for tree languages:

Theorem 4.2 (cf. [7]). A language $L$ is regular iff it is definable in MSO, and both conversions, from automata to formulae and vice versa, are effective. 


\subsection{The Encoding and Preservation of Regularity}

For the rest of this section fix the $K \geq 1$ from above. The first objective is to give the encoding $C: T_{\Sigma, K} \rightarrow \widehat{\Sigma}^{\star}$, where $\$$ is a new symbol and $\widehat{\Sigma}=\Sigma \times\{0,1\} \cup\{\$\}$. For a tree $t \in T_{\Sigma, K}$ of height $m=h(t)$ its encoding $C(t)=\sigma_{0} \sigma_{1} \ldots \sigma_{m}$ is made up of $m+1$ blocks $\sigma_{0}, \ldots, \sigma_{m} \in \widehat{\Sigma}^{K}$ describing the individual levels of $t$. More specifically, $\sigma_{\ell}$ consists of the labels of the $\ell$-th level from left to right, each enriched by a bit stating whether

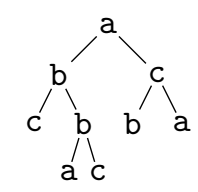

Figure 1. The tree $t_{\mathrm{ex}}$. the corresponding node possesses children, and is padded up to length $K$ by $\$$ symbols. For example, the tree $t_{\mathrm{ex}} \in T_{\{\mathrm{a}, \mathrm{b}, \mathrm{c}\}}$ in Figure 1 on the right satisfies $\varnothing\left(t_{\mathrm{ex}}\right)=4$ and is, under the assumption $K=5$, encoded by the word

$$
C\left(t_{\mathrm{ex}}\right)=\langle\mathrm{a}, 1\rangle \$ \$ \$ \$\langle\mathrm{~b}, 1\rangle\langle\mathrm{c}, 1\rangle \$ \$ \$\langle\mathrm{c}, 0\rangle\langle\mathrm{b}, 1\rangle\langle\mathrm{b}, 0\rangle\langle\mathrm{a}, 0\rangle \$\langle\mathrm{a}, 0\rangle\langle\mathrm{c}, 0\rangle \$ \$ \$ .
$$

Formally, for each $\ell \in[0, m]$ let $u_{\ell, 1}, \ldots, u_{\ell, s_{\ell}}$ be the lexicographic enumeration (w.r.t. $0<1$ ) of $\operatorname{dom}(t) \cap\{0,1\}^{\ell}$. For $r \in\left[1, s_{\ell}\right]$ we let $c_{\ell, r}=1$ if $u_{\ell, r}$ is an inner node, i.e. $u_{\ell, r}\{0,1\} \subseteq \operatorname{dom}(t)$, and $c_{\ell, r}=0$ if $u_{\ell, r}$ is a leaf. Finally, we put

$$
\sigma_{\ell}=\left\langle t\left(u_{\ell, 1}\right), c_{\ell, 1}\right\rangle\left\langle t\left(u_{\ell, 2}\right), c_{\ell, 2}\right\rangle \ldots\left\langle t\left(u_{\ell, s_{\ell}}\right), c_{\ell, s_{\ell}}\right\rangle \$^{K-s_{\ell}} .
$$

The main tool for studying the map $C: T_{\Sigma, K} \rightarrow \widehat{\Sigma}^{\star}$ is the following lemma:

Lemma 4.3. For all $t \in T_{\Sigma, K}$ there is an MSO-interpretation $\left\langle f_{C}, \mathcal{I}_{C}\right\rangle$ of $t$ in $C(t)$ such that $\mathcal{I}_{C}$ does not depend on $t$.

Proof. Observe that for each inner node $u$ of $t$ the children of $u$ are the $(2 s-1)$-th and $2 s$-th node on the next level, where $s$ is the number of inner nodes from left up to $u$ on its level. Formally, for an inner node $u_{\ell, r}$ we have $u_{\ell, r} d=u_{\ell+1,2 s-1+d}$, where $d \in\{0,1\}$ and $s=c_{\ell, 1}+\cdots+c_{\ell, r}$. Based on this observation, one can give an interpretation $\left\langle f_{C}, \mathcal{I}_{C}\right\rangle$ of $t$ in $C(t)$ such that $f_{C}\left(u_{\ell, r}\right)=\ell \cdot K+r$.

As a first consequence, we obtain $t \cong \mathcal{I}_{C}(t)=\mathcal{I}_{C}\left(t^{\prime}\right) \cong t^{\prime}$, and hence $t=t^{\prime}$, for all $t, t^{\prime} \in T_{\Sigma, K}$ with $C(t)=C\left(t^{\prime}\right)$. Thus, the encoding $C$ is one-to-one. The proof of Proposition 4.4 is mainly based on Lemma 4.3 and Lemma 4.5 below.

Proposition 4.4. Let $L \subseteq T_{\Sigma, K}$ be a regular language. Then, the language $C(L) \subseteq \widehat{\Sigma}^{\star}$ is also regular and one can compute a finite automaton recognising $C(L)$ from a tree automaton recognising $L$.

Lemma 4.5. Let $\sigma \in \widehat{\Sigma}^{\star}$. There exists a tree $t \in T_{\Sigma, K}$ with $C(t)=\sigma$ iff $\sigma=\sigma_{0} \sigma_{1} \ldots \sigma_{n}$ for some $n \geq 0$ and $\sigma_{0}, \ldots, \sigma_{n} \in \widehat{\Sigma}^{K}$ satisfying (a) and (b):

(a) $\sigma_{\ell}=\alpha_{\ell, 1} \ldots \alpha_{\ell, s_{\ell}} \$^{K-s_{\ell}}$ for some $s_{\ell} \geq 1$ and $\alpha_{\ell, 1}, \ldots, \alpha_{\ell, s_{\ell}} \in \Sigma \times\{0,1\}$ and for each $\ell \in[0, n]$,

(b) $s_{0}=1, s_{\ell+1}=2 \cdot\left(c_{\ell, 1}+\cdots+c_{\ell, s_{\ell}}\right)$ for $0 \leq \ell<n$, and $c_{m, 1}+\cdots+c_{m, s_{m}}=0$, where $\alpha_{\ell, r}=\left\langle a_{\ell, r}, c_{\ell, r}\right\rangle$. 
Proof. To see that $C(t)$ has the required shape, notice that (b) mainly reflects the relationship between the numbers of nodes on two adjacent levels. Conversely, if $\sigma \in \widehat{\Sigma}^{\star}$ is of the required shape, then there is a tree $t \in T_{\Sigma}$ with $t \cong \mathcal{I}_{C}(\sigma)$ and it turns out that $\varnothing(t) \leq K$ and $C(t)=\sigma 2$

Proof (of Proposition 4.4). Let $\Gamma_{C}$ be an $\mathrm{MSO}_{\mathrm{W}} \widehat{\Sigma}^{- \text {sentence which expresses the }}$ requirement on the shape of $\sigma$ from Lemma 4.5. By Theorem 4.2, there is an MSO $_{\mathrm{T} \Sigma \text {-sentence }} \Phi$ defining $L \subseteq T_{\Sigma, K}$. Then, the $\mathrm{MSO}_{\mathrm{W} \widehat{\Sigma}}$-sentence $\Gamma_{C} \wedge \Phi^{\mathcal{I}_{C}}$ defines $C(L)$ and, again by Theorem 4.2, this language is regular. Finally, all employed constructions are effective.

\subsection{Preservation of Automaticity}

The purpose of this subsection is to complete the proof of Theorem 4.1 .

Proposition 4.6. Let $R \subseteq\left(T_{\Sigma, K}\right)^{n}$ be an automatic relation. Then, the relation $C(R) \subseteq\left(\widehat{\Sigma}^{\star}\right)^{n}$ is also automatic and one can compute a finite automaton recognising $\otimes C(R)$ from a tree automaton recognising $\otimes R$.

Basically, the key idea behind the proof is the same as for Proposition 4.4 though it is more involved. Let $\bar{t}=\left(t_{1}, \ldots, t_{n}\right) \in\left(T_{\Sigma, K}\right)^{n}$. Due to cardinality reasons, $\otimes \bar{t}$ is commonly not directly interpretable in $\otimes C(\bar{t})$ but only in an $n$-fold copy of $\otimes C(\bar{t})$. This is formalised by means of the one-to-one monoid morphism

$$
H:\left(\widehat{\Sigma}_{\square}^{n}\right)^{\star} \rightarrow\left(\widehat{\Sigma}_{\square}^{n}\right)^{\star}, \bar{\alpha}_{1} \ldots \bar{\alpha}_{m} \mapsto \bar{\alpha}_{1}^{n} \ldots \bar{\alpha}_{m}^{n} .
$$

The interpretation of $\otimes \bar{t}$ in $H(\otimes C(\bar{t}))$ embraces two aspects which are better considered separately. Thus, we define an intermediate structure $\amalg \bar{t}$ which extends the disjoint union of the $t_{i}$ 's on domain $\operatorname{dom}(\amalg \bar{t})=\bigcup_{i \in[1, n]}\{i\} \times \operatorname{dom}\left(t_{i}\right)$ by a binary relation $L^{\mathrm{\amalg} \bar{t}}$, relating all $(i, u)$ and $(j, v)$ with $|u|=|v|$, and unary relations $Q_{i}^{\amalg \bar{t}}=\{i\} \times \operatorname{dom}\left(t_{i}\right)$ for each $i \in[1, n]$. Altogether, we give several interpretations whose formulae naturally do not depend on the specific choice of $\bar{t}$. An overview of the whole setting is depicted in Figure 2

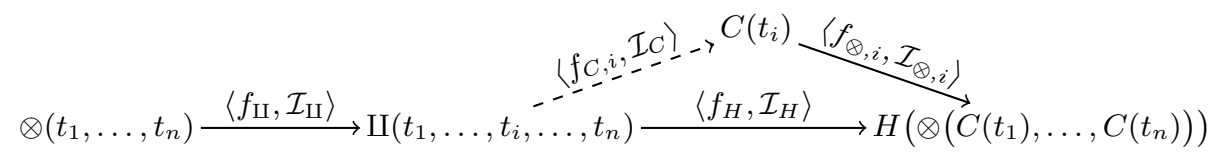

Figure 2. Interpretations involved in proving Proposition 4.6.

The Interpretation $\left\langle f_{\amalg}, \mathcal{I}_{\amalg}\right\rangle$. The main idea is to construct an MSO-formula $E(x, y)$ with $\amalg \bar{t} \models E((i, u),(j, v))$ iff $u=v$. To achieve this, consider for each $(i, u) \in \operatorname{dom}(\amalg \bar{t})$ the set $\operatorname{Pre}(i, u)$ of all $\left(i, u^{\prime}\right) \in \operatorname{dom}(\amalg \bar{t})$ where $u^{\prime}$ is a prefix of $u$. For $(i, u),(j, v) \in \operatorname{dom}(\amalg \bar{t})$ we have $u=v$ iff $|u|=|v|$ and for all $\left(i, u^{\prime}\right) \in$

\footnotetext{
${ }^{2}$ More details on this can be found in Appendix B.2.
} 
$\operatorname{Pre}(i, u)$ and $\left(j, v^{\prime}\right) \in \operatorname{Pre}(j, v)$ with $\left|u^{\prime}\right|=\left|v^{\prime}\right|>0$ the last symbols of $u^{\prime}$ and $v^{\prime}$ coincide. Since the set $\operatorname{Pre}((i, u), X)$ is definable in MSO, we can express this characterisation in MSO as well. Heavily using the resulting formula $E$, one can construct an interpretation $\left\langle f_{\amalg}, \mathcal{I}_{\amalg}\right\rangle$ of $\otimes \bar{t}$ in $\amalg \bar{t}$ such that $f_{\amalg}(u)=(i, u)$, where $i$ is minimal with $u \in \operatorname{dom}\left(t_{i}\right)$.

The Interpretations $\left\langle f_{\otimes, i}, \mathcal{I}_{\otimes, i}\right\rangle$. For all $i \in[1, n]$ and $\bar{w} \in\left(\widehat{\Sigma}^{\star}\right)^{n}$ one can easily give an interpretation $\left\langle f_{\otimes, i}, \mathcal{I}_{\otimes, i}\right\rangle$ of $w_{i}$ in $H(\otimes \bar{w})$ such that $f_{\otimes, i}(p)=(p-1) \cdot n+i$.

The Interpretation $\left\langle f_{H}, \mathcal{I}_{H}\right\rangle$. For $i \in[1, n]$ let $\left\langle f_{C, i}, \mathcal{I}_{C}\right\rangle$ be the interpretation of $t_{i}$ in $C\left(t_{i}\right)$ from Lemma 4.3 Since the $f_{\otimes, i}$ 's have mutually disjoint images, the map $f_{H}: \operatorname{dom}(\amalg \bar{t}) \rightarrow \operatorname{dom}(H(\otimes C(\bar{t})))$ with $f_{H}(i, u)=f_{\otimes, i}\left(f_{C, i}(u)\right)$ is oneto-one. For $(i, u) \in \operatorname{dom}(\amalg \bar{t})$ we get $|u| \cdot K<f_{C, i}(u) \leq(|u|+1) \cdot K$ and hence

$$
|u| \cdot K \cdot n<f_{H}(i, u) \leq(|u|+1) \cdot K \cdot n .
$$

Exploiting this observation for the formula $L^{\mathcal{I}_{H}}$ and using $\mathcal{I}_{C}$ and $\mathcal{I}_{\otimes, i}$, one can construct formulae $\mathcal{I}_{H}$ such that $\left\langle f_{H}, \mathcal{I}_{H}\right\rangle$ is an interpretation of $\amalg \bar{t}$ in $H(\otimes C(\bar{t}))$.

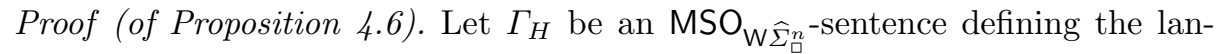
guage $H\left(\otimes\left(\widehat{\Sigma}^{\star}\right)^{n}\right) \subseteq\left(\widehat{\Sigma}_{\square}^{n}\right)^{\star}$. If $\Phi$ defines $\otimes R$, then

$$
\Gamma_{H} \wedge \bigwedge_{i \in[1, n]} \Gamma_{C}^{\mathcal{I}_{\otimes, i}} \wedge\left(\Phi^{\mathcal{I}_{\amalg}}\right)^{\mathcal{I}_{H}}
$$

defines $H(\otimes C(R))$. Since $H$ is a one-to-one monoid morphism, $\otimes C(R)$ is regular as well. Finally, all employed constructions are effective.

\section{Fat Tree Automatic Ordinals Are Not Word Automatic}

The goal of this section is to give the last missing piece for the proof of Theorem 1.1, namely the following theorem:

Theorem 5.1. Let $\mathfrak{L}$ be a tree automatic scattered linear ordering such that $\operatorname{dom}(\mathfrak{L})$ is fat. Then, $\mathfrak{L}$ is not word automatic.

The theorem below states the necessary condition on word automatic linear orderings we use to show non-automaticity:

Theorem 5.2 (Khoussainov, Rubin, Stephan [5]). If $\mathfrak{L}$ is a word automatic linear ordering, then its FC-rank is finite.

Actually, we do not need any details on the $F C$-rank (finite condensation rank) besides the fact that every scattered linear ordering $\mathfrak{L}$, having the property that for each $r \geq 1$ at least one linear ordering from

$$
\mathcal{N}_{r}=\left\{\mathfrak{A}_{1} \cdot \mathfrak{A}_{2} \cdots \mathfrak{A}_{r} \mid \mathfrak{A}_{1}, \ldots, \mathfrak{A}_{r} \in\{(\mathbb{N} ;<),(\mathbb{N} ;>)\}\right\}
$$

can be embedded into $\mathfrak{L}$, has infinite $F C$-rank. The main idea of the proof is as follows: 
Lemma 5.3. Let $\mathfrak{L}=(L ;<)$ be a tree automatic scattered linear ordering, $\left(\mathcal{A} ; \mathcal{A}_{<}\right)$an automatic presentation of $\mathfrak{L}, n$ the number of states of $\mathcal{A}$, and $r \geq 1$. If there exists some tree $t \in L$ with $\varnothing(t) \geq r \cdot 2^{n}$, then there are infinite linear orderings $\mathfrak{A}_{1}, \ldots, \mathfrak{A}_{r}$ such that $\mathfrak{A}_{1} \cdot \mathfrak{A}_{2} \cdots \mathfrak{A}_{r}$ can be embedded into $\mathfrak{L}$.

For any linear ordering $\mathfrak{A}$ and all $a_{1}, a_{2} \in \operatorname{dom}(\mathfrak{A})$ we define $\operatorname{cmp}_{\mathfrak{A}}\left(a_{1}, a_{2}\right) \in$ $\{-1,0,1\}$ to be -1 if $a_{1}<^{\mathfrak{A}} a_{2}, 0$ if $a_{1}=a_{2}$, and 1 if $a_{2}<^{\mathfrak{A}} a_{1}$. To simplify notation, we put $\llbracket s, t \rrbracket_{<}=\mathcal{A}_{<}(\otimes(s, t))$ for all $s, t \in T_{\Sigma}$. Moreover, we assume w.l.o.g. that from $\llbracket s, t \rrbracket_{<}$one can deduce whether $s=t$ holds true. Then, $\operatorname{cmp}_{\mathfrak{L}}(s, t)$ is determined by $\llbracket s, t \rrbracket_{<}$for all $s, t \in L$, i.e., there is a map $f$ from the state set of $\mathcal{A}_{<}$to $\{-1,0,1\}$ such that $\operatorname{cmp}_{\mathfrak{L}}(s, t)=f\left(\llbracket s, t \rrbracket_{<}\right)$for all $s, t \in L$.

Proof. Let $\mathfrak{T} \in L$ be a tree and $\ell \geq n$ such that $\left|\operatorname{dom}(\mathfrak{T}) \cap\{0,1\}^{\ell}\right| \geq r \cdot 2^{n}$. Thus, there exist at least $r$ mutually distinct $u \in \operatorname{dom}(\mathfrak{T}) \cap\{0,1\}^{\ell-n}$ for which there is a $v \in\{0,1\}^{n}$ with $u v \in \operatorname{dom}(\mathfrak{T})$, say $u_{1}, \ldots, u_{r}$. For $\bar{t}=\left(t_{1}, \ldots, t_{r}\right) \in\left(T_{\Sigma}\right)^{r}$ let $\mathfrak{T}[t] \in T_{\Sigma}$ be the tree obtained from $\mathfrak{T}$ by replacing for each $i \in[1, r]$ the subtree rooted at $u_{i}$ with $t_{i}$. Then, $\mathcal{A}(\mathfrak{T}[t])$ is determined by the $r$ states $\mathcal{A}\left(t_{1}\right), \ldots, \mathcal{A}\left(t_{r}\right)$ for all $\bar{t} \in\left(T_{\Sigma}\right)^{r}$. Moreover, for $\bar{s} \in\left(T_{\Sigma}\right)^{r}$ the tree $\otimes(\mathfrak{T}[\bar{s}], \mathfrak{T}[\bar{t}])$ is obtained from $\otimes(\mathfrak{T}, \mathfrak{T})$ by replacing for each $i \in[1, r]$ the subtree rooted at $u_{i}$ with $\otimes\left(s_{i}, t_{i}\right)$. Consequently, $\llbracket \mathfrak{T}[\bar{s}], \mathfrak{T}[\bar{t}] \rrbracket_{<}$is determined by the $r$ states $\llbracket s_{1}, t_{1} \rrbracket_{<}, \ldots, \llbracket s_{r}, t_{r} \rrbracket_{<}$ for all $\bar{s}, \bar{t} \in\left(T_{\Sigma}\right)^{r}$.

Observe that $h\left(\mathfrak{T} \mid u_{i}\right) \geq n$ for each $i \in[1, r]$. Therefore, by Lemma 3.3 and Ramsey's theorem for infinite, undirected, finitely coloured graphs, there exists an infinite set $A_{i} \subseteq T_{\Sigma}$ of trees $t \in T_{\Sigma}$ with $\mathcal{A}(t)=\mathcal{A}\left(\mathfrak{T}\left\lceil u_{i}\right)\right.$ such that

$$
c(s, t)=\left\{\llbracket s, s \rrbracket_{<,} \llbracket t, t \rrbracket_{<,} \llbracket s, t \rrbracket_{<,}, \llbracket t, s \rrbracket_{<}\right\}
$$

is the same set $Q_{i}$ for all distinct $s, t \in A_{i}$. It turns out that $Q_{i}$ has exactly three elements and $\llbracket s, s \rrbracket_{<}=\llbracket t, t \rrbracket_{<}$for all $s, t \in A_{i}$.

Now, put $A=A_{1} \times \cdots \times A_{r}$. For each $\bar{t} \in A$ we have $\mathcal{A}(\mathfrak{T}[\bar{t}])=\mathcal{A}(\mathfrak{T})$ and hence $\mathfrak{T}[\bar{t}] \in L$. We define a linear ordering $\mathfrak{A}=\left(A ;<^{\mathfrak{A}}\right)$ by $\bar{s}<{ }^{\mathfrak{A}} \bar{t}$ iff $\mathfrak{T}[\bar{s}]<\mathfrak{T}[\bar{t}]$. By definition, $\mathfrak{A}$ can be embedded into $\mathfrak{L}$.

For $i \in[1, r], \bar{a} \in A$, and $t \in A_{i}$ we let $\bar{a}_{i / t} \in A$ be the tuple $\bar{a}$ with the $i$-th component replaced by $t$. Then, for all $\bar{a}, \bar{b}$ and $s, t \in A_{i}$ we obtain $\llbracket \mathfrak{T}\left[\bar{a}_{i / s}\right], \mathfrak{T}\left[\bar{a}_{i / t}\right] \rrbracket_{<}=\llbracket \mathfrak{T}\left[\bar{b}_{i / s}\right], \mathfrak{T}\left[\bar{b}_{i / t}\right] \rrbracket_{<}$and hence $a_{i / s}<{ }^{\mathfrak{A}} a_{i / t}$ iff $b_{i / s}<\mathfrak{A} b_{i / t}$. Thus, defining a linear ordering $\mathfrak{A}_{i}=\left(A_{i} ;<^{\mathfrak{A}_{i}}\right)$ by $s<<^{\mathfrak{A}_{i}} t$ iff $\bar{a}_{i / s}<{ }^{\mathfrak{A}} \bar{a}_{i / t}$ is independent from the specific choice of $\bar{a} \in A$. Clearly, $\operatorname{cmp}_{\mathfrak{A}_{i}}(s, t)$ is determined by $\llbracket s, t \rrbracket_{<}$for all $s, t \in A_{i}$. Since $Q_{i}$ contains exactly three elements, $\llbracket s, t \rrbracket_{<}$is determined by $\operatorname{cmp}_{\mathfrak{A}_{i}}(s, t)$ for all $s, t \in A_{i}$ as well. Hence, the linear orderings $\mathfrak{A}$ and $\mathfrak{A}_{1}, \ldots, \mathfrak{A}_{r}$ satisfy the condition of Lemma 5.4 below and consequently $\mathfrak{A}_{\pi(1)} \cdots \mathfrak{A}_{\pi(r)}$ can be embedded into $\mathfrak{L}$.

Lemma 5.4. Let $\mathfrak{A}$ and $\mathfrak{A}_{1}, \ldots, \mathfrak{A}_{r}$ be infinite linear orderings with $\operatorname{dom}(\mathfrak{A})=$ $\operatorname{dom}\left(\mathfrak{A}_{1}\right) \times \cdots \times \operatorname{dom}\left(\mathfrak{A}_{r}\right)$ and satisfying the following two conditions:

(1) $\operatorname{cmp}_{\mathfrak{A}_{\mathfrak{A}}}(\bar{a}, \bar{b})$ is determined by $\operatorname{cmp}_{\mathfrak{A}_{1}}\left(a_{1}, b_{1}\right), \ldots, \operatorname{cmp}_{\mathfrak{A}_{r}}\left(a_{r}, b_{r}\right)$ for all $\bar{a}, \bar{b} \in A$,

(2) if $\bar{a}, \bar{b} \in A$ differ only in the $i$-th component, then $\operatorname{cmp}_{\mathfrak{A}}(\bar{a}, \bar{b})=\operatorname{cmp}_{\mathfrak{A}_{i}}\left(a_{i}, b_{i}\right)$. 
Then, there exists a permutation $\pi$ of $\{1, \ldots, r\}$ such that $\mathfrak{A}$ is isomorphic to $\mathfrak{A}_{\pi(1)} \cdot \mathfrak{A}_{\pi(2)} \cdots \mathfrak{A}_{\pi(r)}$.

Finally, we are in a position to prove Theorem 5.1.

Proof (of Theorem 5.1). Let $\left(\mathcal{A} ; \mathcal{A}_{<}\right)$be an automatic presentation of $\mathfrak{L}$ and $n$ the number of states of $\mathcal{A}$. Since $\operatorname{dom}(\mathfrak{L})$ is fat, for any $r \geq 1$ there is a $t \in \operatorname{dom}(\mathfrak{L})$ with $\varnothing(t) \geq r \cdot 2^{n}$. Let $\mathfrak{A}_{1}, \ldots, \mathfrak{A}_{r}$ be the infinite linear orderings from Lemma 5.3 , For each $i \in[1, r]$ some $\mathfrak{B}_{i} \in\{(\mathbb{N} ;<),(\mathbb{N} ;>)\}$ can be embedded into $\mathfrak{A}_{i}$. Then, $\mathfrak{B}_{1} \cdot \mathfrak{B}_{2} \cdots \mathfrak{B}_{r} \in \mathcal{N}_{r}$ can be embedded into $\mathfrak{A}_{1} \cdot \mathfrak{A}_{2} \cdots \mathfrak{A}_{r}$ and consequently into $\mathfrak{L}$. Hence, $\mathfrak{L}$ has infinite $F C$-rank and is, by Theorem [5.2, not word automatic.

\section{Conclusions}

Altogether, we proved that is decidable whether a given tree automatic scattered linear ordering is already word automatic. Taking a closer look at the proof reveals that the problem is solvable nondeterministically in logarithmic space, provided the tree automaton recognising the domain is reduced.

The restriction to scattered linear orderings naturally rises the question whether this result holds true for general linear orderings. Unfortunately, this problem cannot be solved by means of our technique since the ordering $(\mathbb{Q} ;<)$ of the rationals admits a word automatic as well as a fat tree automatic presentation. As the Boolean algebra of finite and co-finite subsets of $\mathbb{N}$ shares this feature, the same pertains to an analogue of Theorem 1.1 for Boolean algebras. In spite of that, we suggest trying to apply the technique to other classes of structures, such as groups, for which a necessary condition on its automatic members is known.

Finally, Theorem 1.1 provides a decidable characterisation of all tree automatic ordinals $\alpha \geq \omega^{\omega}$. Finding such a characterisation for each $\omega^{\omega^{k}}$ with $k \in \mathbb{N}$ possibly turns out to be the main ingredient for showing that the isomorphism problem for tree automatic ordinals is decidable.

\section{References}

1. Bárány, V., Grädel, E., Rubin, S.: Automata-based presentations of infinite structures. In Esparza, J., Michaux, C., Steinhorn, C., eds.: Finite and Algorithmic Model Theory. Cambridge University Press (2011) 1-76

2. Blumensath, A.: Automatic structures. Diploma thesis, RWTH Aachen (1999)

3. Gécseg, F., Steinby, M.: Tree languages. In Rozenberg, G., Salomaa, A., eds.: Handbook of Formal Languages. Volume 3. Springer (1997) 1-68

4. Khoussainov, B., Nerode, A.: Automatic presentations of structures. In: LCC 1994. Volume 960 of LNCS, Springer (1995) 367-392

5. Khoussainov, B., Rubin, S., Stephan, F.: On automatic partial orders. In: LICS 2003. (2003) 168-177

6. Rubin, S.: Automata presenting structures: A survey of the finite string case. Bulletin of Symbolic Logic 14(2) (2008) 169-209

7. Thomas, W.: Languages, automata, and logic. In Rozenberg, G., Salomaa, A., eds.: Handbook of Formal Languages. Volume 3. Springer (1997) 384-455 


\section{A Proofs of Lemmas 3.3 and 3.4}

Recall that we fixed a reduced tree automaton $\mathcal{A}=(Q, \iota, \delta, F)$ and defined the graph $G_{\mathcal{A}}=\left(Q, E_{\mathcal{A}}\right)$ by

$$
(p, q) \in E_{\mathcal{A}} \quad \text { iff } \quad \exists a \in \Sigma, r \in Q: \delta(a, p, r)=q \text { or } \delta(a, r, p)=q .
$$

An edge $(p, q) \in E_{\mathcal{A}}$ was called special if in Eq. (1) the state $r \in Q$ can be chosen such that it satisfies the conditions of Lemma 3.3. Moreover, we denote the prefix relation on $\{0,1\}^{\star}$ by $\preceq$, i.e., $u \preceq v$ if there is some $w \in\{0,1\}^{\star}$ such that $u w=v$.

To simplify notation, we put $t[u]=\mathcal{A}(t \mid u)$ for each $t \in T_{\Sigma}$ and $u \in \operatorname{dom}(t)$. In particular, $t[\varepsilon]=\mathcal{A}(t)$. For all $u \in \operatorname{dom}(t)$ with $u 0, u 1 \in \operatorname{dom}(t)$ we have $\delta(t(u), t[u 0], t[u 1])=t[u]$ and hence $(t[u d], t[u]) \in E_{\mathcal{A}}$ for both $d=0$ and $d=1$. We denote these edges by $t[u d, u]$. Applying this argument repeatedly, for all $u, v \in \operatorname{dom}(t)$ with $u \preceq v$ we obtain a path from $t[v]$ to $t[u]$ of length ${ }^{3}|v|-|u|$ in $G_{\mathcal{A}}$, which we denote by $t[v, u]$.

Conversely, let $(p, q) \in E_{\mathcal{A}}$ be an edge in $G_{\mathcal{A}}$ and $t \in T_{\Sigma}$ a tree with $\mathcal{A}(t)=p$. Then, there are $a \in \Sigma$ and $r \in Q$ such that, w.l.o.g., $\delta(a, p, r)=q$. Moreover, there is a tree $s \in T_{\Sigma}$ with $\mathcal{A}(s)=r$. Then, the unique tree $t^{\prime} \in T_{\Sigma}$ with $t^{\prime}(\varepsilon)=a, t^{\prime}\left\lceil 0=t\right.$, and $t^{\prime}\left\lceil 1=s\right.$ satisfies $\mathcal{A}\left(t^{\prime}\right)=q$ and $t^{\prime}\lceil 0=t$. Applying this argument repeatedly, we obtain for each path from $p$ to $q$ of length $m$ in $G_{\mathcal{A}}$ and any tree $t \in T_{\Sigma}$ with $\mathcal{A}(t)=p$ another tree $t^{\prime} \in T_{\Sigma}$ and a position $u \in \operatorname{dom}\left(t^{\prime}\right)$ such that $\mathcal{A}\left(t^{\prime}\right)=q,|u|=m$, and $t^{\prime}\lceil u=t$.

Lemma 3.3 For every $q \in Q$ the following are equivalent:

(1) there are infinitely many $t \in T_{\Sigma}$ satisfying $\mathcal{A}(t)=q$,

(2) there is a tree $t \in T_{\Sigma}$ satisfying $h(t) \geq n$ and $\mathcal{A}(t)=q$, where $n=|Q|$,

(3) $G_{\mathcal{A}}$ contains a cycle from which $q$ is reachable.

Proof. Trivially, (1) implies (2). It remains to show that (2) implies (3) and (3) implies (1).

To (2) implies (3). Let $t \in T_{\Sigma}$ be a tree with $h(t) \geq n$ and $\mathcal{A}(t)=q$. Consider some $u \in \operatorname{dom}(t)$ such that $|u|=n$. Then, $t[u, \varepsilon]$ is a path of length $n$ ending in $q$. Due to the pigeonhole principle, this path contains a cycle.

To (3) implies (1). It suffices to show that for each $m \geq 0$ there is a tree $t \in T_{\Sigma}$ with $\mathcal{A}(t)=q$ and $h(t) \geq m$. Thus, consider some $m \geq 0$. There is a path of length $m$ ending in $q$. Let $p \in Q$ be the first state of this path and $s \in T_{\Sigma}$ a tree with $\mathcal{A}(s)=p$. Then, there are a tree $t \in T_{\Sigma}$ and $u \in \operatorname{dom}(t)$ with $\mathcal{A}(t)=q$, $|u|=m$, and $t\lceil u=s$. In particular, $h(t) \geq m$.

\footnotetext{
${ }^{3}$ The length of a path is the number of its edges.
} 
Lemma 3.4 The following are equivalent:

(1) the tree language $L$ recognised by $\mathcal{A}$ is fat,

(2) there is a tree $t \in L$ satisfying $\varnothing(t)>2^{n-1}$, where $n=|Q|$,

(3) $G_{\mathcal{A}}$ contains a cycle including a special edge and from which $F$ is reachable.

Proof. Trivially, (1) implies (2). It remains to show that (2) implies (3) and (3) implies (1).

To (2) implies (3). Using induction on $m \geq 0$ we show the following: For every tree $t \in T_{\Sigma}$ with $\varnothing(t)>2^{m-1}$ and $\left|Q_{t}\right| \leq m$, where

$$
Q_{t}=\{t[u] \mid u \in \operatorname{dom}(r)\},
$$

there are $u_{1}, u_{2} \in \operatorname{dom}(t)$ such that $u_{1} \prec u_{2}$ and $t\left[u_{2}, u_{1}\right]$ is a cycle containing a special edge.

For $m=0$ there is nothing to show. Thus, let $m>0$. Consider an $\ell \geq 0$ such that $|U|>2^{m-1}$ for $U=\operatorname{dom}(t) \cap\{0,1\}^{\ell}$. Let $u \in \operatorname{dom}(t)$ be the longest common prefix of all positions in $U$. Clearly, $\ell \geq|u|+m$. There are two cases:

1. There is a $v \in \operatorname{dom}(t)$ with $u \prec v$ and $t[u]=t[v]$. W.l.o.g., we assume $u 0 \preceq v$. By the choice of $u$, there is some $w \in U$ with $u 1 \preceq w$. The path $t[w, u]$ has length $\ell-|u| \geq m$ and hence contains a cycle. Since $t[u 1]$ lies on or after this cycle, i.e., $t[u 1]$ satisfies condition (3) of Lemma 3.3. Thus, the edge $t[u 0, u] \in E_{\mathcal{A}}$, which is contained in the cycle $t[v, u]$, is special.

2. There is no $v \in \operatorname{dom}(t)$ with $u \prec v$ and $t[u]=t[v]$. In particular, $2 \leq\left|Q_{t}\right| \leq$ $m$. Since $\varnothing(t \mid u) \geq|U|>2^{m-1}$, we have $\varnothing\left(t\lceil u 0)>2^{m-2}\right.$ or $\varnothing\left(t\lceil u 1)>2^{m-2}\right.$. W.l.o.g., assume $\varnothing(s)>2^{m-2}$ for $s=t\left\lceil u 0\right.$. We have $t[u] \notin Q_{s}$ and hence $\left|Q_{s}\right|<\left|Q_{t}\right| \leq m$. By the induction hypothesis, there are $v, w \in \operatorname{dom}(s)$ such that $v \prec w$ and $s[w, v]$ is a cycle containing a special edge. The claim follows from $u 0 v \prec u 0 w$ and $t[u 0 w, u 0 v]=s[w, v]$.

Finally, consider some $t \in L(\mathcal{A})$ with $\varnothing(t)>2^{n-1}$. Obviously, $\left|Q_{t}\right| \leq|Q|=n$. From the cycle $t\left[u_{2}, u_{1}\right]$ we can reach a state from $F$ along the path $t\left[u_{1}, \varepsilon\right]$.

To (3) implies (1). Using induction on $m \geq 0$ we show that if there is a path containing $m$ special edges and which ends in some $q \in Q$, then there is a tree $t \in T_{\Sigma}$ with $\mathcal{A}(t)=q$ and $\varnothing(t)>m$. Due to the cycle there are paths containing arbitrarily many special edges and which end in $F$. Thus, condition (1) will follow.

For $m=0$ any tree $t \in T_{\Sigma}$ with $\mathcal{A}(t)=q$ trivially satisfies $\varnothing(t)>0$. Thus, consider $m>0$. Let $(p, r) \in E_{\mathcal{A}}$ be the last special edge in the path. By the induction hypothesis, there is a tree $s \in T_{\Sigma}$ with $\mathcal{A}(s)=p$ and $\varnothing(s)>m-1$. Let $\ell \geq 0$ be such that $\left|\operatorname{dom}(s) \cap\{0,1\}^{\ell}\right|=\varnothing(s)$. Moreover, there are $a \in \Sigma$ and $p^{\prime} \in Q$ such that, w.l.o.g., $\delta\left(a, p, p^{\prime}\right)=r$ and $p^{\prime}$ satisfies the conditions of Lemma 3.3. Thus, there is a tree $s^{\prime} \in T_{\Sigma}$ such that $h\left(s^{\prime}\right) \geq \ell$. Then, the unique tree $t^{\prime} \in T_{\Sigma}$ with $t^{\prime}(\varepsilon)=a, t^{\prime}\left\lceil 0=s\right.$, and $t^{\prime}\left\lceil 1=s^{\prime}\right.$ satisfies $\mathcal{A}\left(t^{\prime}\right)=r$. Since there 
is a path from $r$ to $q$, say it has $n$ edges, there are $t \in T_{\Sigma}$ and $u \in \operatorname{dom}(t)$ such that $\mathcal{A}(t)=q,|u|=n$, and $t\left\lceil u=t^{\prime}\right.$. From the construction of $t$ we obtain

$$
\begin{aligned}
\operatorname{dom}(t) \cap\{0,1\}^{n+1+\ell} \supseteq u( & \left.\operatorname{dom}\left(t^{\prime}\right) \cap\{0,1\}^{1+\ell}\right) \\
& =u 0\left(\operatorname{dom}(s) \cap\{0,1\}^{\ell}\right) \cup u 1\left(\operatorname{dom}\left(s^{\prime}\right) \cap\{0,1\}^{\ell}\right) .
\end{aligned}
$$

Since the union on the right hand side is disjoint and the set $\operatorname{dom}\left(s^{\prime}\right) \cap\{0,1\}^{\ell}$ is not empty, we have

$$
\left|\operatorname{dom}(t) \cap\{0,1\}^{n+1+\ell}\right| \geq\left|\operatorname{dom}(s) \cap\{0,1\}^{\ell}\right|+\mid\left(\operatorname{dom}\left(s^{\prime}\right) \cap\{0,1\}^{\ell} \mid>m,\right.
$$

i.e., $\varnothing(t)>m$. 


\section{B Interpretations and Formulae from Section 4}

\section{B.1 The Interpretation $\left\langle f_{C}, \mathcal{I}_{C}\right\rangle$}

Lemma 4.3 For all $t \in T_{\Sigma, K}$ there is an MSO-interpretation $\left\langle f_{C}, \mathcal{I}_{C}\right\rangle$ of $t$ in $C(t)$ such that $\mathcal{I}_{C}$ does not depend on $t$.

The formulae $\mathcal{I}_{C}=\left(\Delta_{C} ;\left(S_{d}^{\mathcal{I}_{C}}\right)_{d \in\{0,1\}},\left(P_{a}^{\mathcal{I}_{C}}\right)_{a \in \Sigma}\right)$ are as follows:

$$
\begin{aligned}
& \Delta_{C}(x)=\neg P_{\$}(x) \\
& S_{d}^{\mathcal{I}_{C}}(x, y)=\bigvee_{a \in \Sigma} P_{(a, 1)}(x) \wedge \Delta_{C}(y) \wedge \exists z\left(\varphi(x, z) \wedge \bigvee_{1 \leq s \leq \frac{K}{2}} \psi_{d, s}(x, y, z)\right) \\
& P_{a}^{\mathcal{I}_{C}}(x)=\Delta_{C}(x) \wedge\left(P_{(a, 0)}(x) \vee P_{(a, 1)}(x)\right) \\
& \varphi(x, z)=z \equiv 1(\bmod K) \wedge z \leq x<z+K \\
& \psi_{d, s}(x, y, z)=\exists^{=s} z^{\prime}\left(z \leq z^{\prime} \leq x \wedge \bigvee_{a \in \Sigma} P_{(a, 1)}\left(z^{\prime}\right)\right) \wedge y=z+K+2 s-2+d \\
& \quad\left(1 \leq s \leq \frac{K}{2}\right)
\end{aligned}
$$

\section{B.2 Proof of Lemma 4.5}

Lemma 4.5 Let $\sigma \in \widehat{\Sigma}^{\star}$. There exists a tree $t \in T_{\Sigma, K}$ with $C(t)=\sigma$ iff $\sigma=$ $\sigma_{0} \sigma_{1} \ldots \sigma_{n}$ for some $n \geq 0$ and $\sigma_{0}, \ldots, \sigma_{n} \in \widehat{\Sigma}^{K}$ satisfying (a) and (b):

(a) $\sigma_{\ell}=\alpha_{\ell, 1} \ldots \alpha_{\ell, s_{\ell}} \$^{K-s_{\ell}}$ for some $s_{\ell} \geq 1$ and $\alpha_{\ell, 1}, \ldots, \alpha_{\ell, s_{\ell}} \in \Sigma \times\{0,1\}$ and for each $\ell \in[0, n]$,

(b) $s_{0}=1, s_{\ell+1}=2 \cdot\left(c_{\ell, 1}+\cdots+c_{\ell, s_{\ell}}\right)$ for $0 \leq \ell<n$, and $c_{m, 1}+\cdots+c_{m, s_{m}}=0$, where $\alpha_{\ell, r}=\left\langle a_{\ell, r}, c_{\ell, r}\right\rangle$.

Proof. To see that $C(t)$ has the required shape, choose $n=m, \sigma_{\ell}$ and $s_{\ell}$ as in the construction of $C(t)$, and $\alpha_{\ell, r}=\left\langle t\left(u_{\ell, r}\right), c_{\ell, r}\right\rangle$. Then, condition (a) is trivially met, whereas (b) is satisfied since each tree has exactly one node on the zeroth level, on each other level twice as many nodes as inner nodes on the previous level, and no inner nodes on the last level.

Conversely, consider some $\sigma=\alpha_{1} \ldots \alpha_{(n+1) \cdot K} \in \widehat{\Sigma}^{\star}$ of the required shape. Let $\mathfrak{T}=\mathcal{I}_{C}(\sigma)$ and $T=\operatorname{dom}(\mathfrak{T})$. First, we observe that

$$
T=\left\{\ell \cdot K+r \mid \ell \in[0, n], r \in\left[1, s_{\ell}\right]\right\}
$$

and put $\left(a_{p}, c_{p}\right)=\alpha_{p}$ for all $p \in T$. For $p=\ell \cdot K+r, q \in T$ and $d \in\{0,1\}$ with $\ell \in[0, n]$ and $r \in\left[1, s_{\ell}\right]$ we have $(p, q) \in S_{d}^{\mathfrak{T}}$ iff $c_{p}=1$ and $q=(\ell+1) \cdot K+2 s-1+d$, where $s=c_{\ell \cdot K+1}+\cdots+c_{p}$. Notice that this reflects the introductory observation in the proof of Lemma 4.3

Second, we construct a map $f: T \rightarrow\{0,1\}^{\star}$. We define the value $f(q)$ by induction on $q \in T$. The resulting map will satisfy $|f(q)|=\ell$ for all $q \in T$ with $\ell \cdot K<q \leq(\ell+1) \cdot K$. The first condition of (b) yields $q=1$ or $q>K$. We put $f(1)=\varepsilon$. For $q>K$ there are unique $\ell, r \in \mathbb{N}$ with $0 \leq \ell<n$ and $1 \leq s \leq \frac{s_{\ell+1}}{2}$, and $d \in\{0,1\}$ such that $q=(\ell+1) \cdot K+2 s-1+d$. From the 
second condition of (b) we conclude $s \leq c_{\ell \cdot K+1}+\ldots+c_{\ell \cdot K+s_{\ell}}$. Thus, there is a least $p \in\left[\ell \cdot K+1, \ell \cdot K+s_{\ell}\right]$ such that $s=c_{\ell \cdot K+1}+\cdots+c_{p}$ and the minimality implies $c_{p}=1$. Since $p<q$, we are allowed to put $f(q)=f(p) d$.

A simple but tedious inspection of this construction shows for all $p, q \in T$ that $p<q$ iff $f(p)<_{\text {llex }} f(q)$, where $<_{\text {llex }}$ is the length-lexicographic order on $\{0,1\}^{\star}$. In particular, $f$ is one-to-one. Obviously, the set $D=f(T)$ is non-empty and finite. Due to the construction of $f$ it is also prefix-closed. For $u 0 \in D$ we have $f^{-1}(u 0)+1 \in T$ and $u 1=f\left(f^{-1}(u 0)+1\right) \in D$. Similarly, $u 1 \in D$ implies $u 0 \in D$. Thus, $D$ is a tree domain. We define a tree $t \in T_{\Sigma}$ by $\operatorname{dom}(t)=D$ and $t(u)=a_{f-1}(u)$. It turns out that $f$ induces an isomorphism $f: \mathfrak{T} \rightarrow t$ between T $\Sigma$-structures.

From the earlier remark on $|f(q)|$ we conclude $\varnothing(t) \leq K$. Moreover, $h(t)=n$ and $f(\ell \cdot K+1), \ldots, f\left(\ell \cdot K+s_{\ell}\right)$ is the lexicographic enumeration of $\operatorname{dom}(t) \cap$ $\{0,1\}^{\ell}$ for all $0 \leq \ell \leq n$. For $p \in T$ with $p \leq n \cdot K$ the specific choice of $f$ yields $c_{p}=1$ iff $f(p)\{0,1\} \subseteq \operatorname{dom}(t)$. For $p>n \cdot K$ this also holds true since the third condition of (b) implies $c_{p}=0$ and $|f(p) d|>n$ implies $f(p) d \notin \operatorname{dom}(t)$ for $d \in\{0,1\}$. Altogether, we obtain $C(t)=\sigma$.

\section{B.3 The Sentence $\Gamma_{C}$}

The sentence $\Gamma_{C}$ is defined as follows:

$$
\begin{aligned}
\Gamma_{C} & =\Phi_{1} \wedge \Phi_{2} \wedge \Phi_{3} \wedge \Phi_{4} \wedge \Phi_{5} \\
\Phi_{1} & =\exists x(\forall y(y \leq x) \wedge x \equiv K(\bmod K)) \\
\Phi_{2} & =\forall x(x \equiv 1(\bmod K) \rightarrow \exists y \varphi(x, y)) \\
\Phi_{3} & =\exists x(x=1 \wedge \varphi(x, x)) \\
\Phi_{4} & =\forall x_{1} \forall x_{2}\left(x_{1} \equiv 1(\bmod K) \wedge x_{2}=x_{1}+K \rightarrow \bigvee_{1 \leq s \leq \frac{K}{2}} \psi_{s}\left(x_{1}, x_{2}\right)\right) \\
\Phi_{5} & =\exists x\left(x \equiv 1(\bmod K) \wedge \neg \exists z(z=x+K) \wedge \exists y\left(\varphi(x, y) \wedge \chi_{0}(x, y)\right)\right) \\
\varphi(x, y) & =x \leq y<x+K \wedge \forall z\left(x \leq z<x+K \rightarrow\left(P_{\$}(z) \leftrightarrow y<z\right)\right) \\
\psi_{s}(x) & =\exists y_{1} \exists y_{2}\left(\varphi\left(x_{1}, y_{1}\right) \wedge \varphi\left(x_{2}, y_{2}\right) \wedge \chi_{s}\left(x_{1}, y_{1}\right) \wedge y_{2}=x_{2}+2 s-1\right) \\
\chi_{s}(x, y) & =\exists=s z\left(x \leq z \leq y \wedge \bigvee_{a \in \Sigma} P_{(a, 1)}(z)\right) \quad\left(1 \leq s \leq \frac{K}{2}\right)
\end{aligned}
$$

The purpose of the formulae $\Phi_{i}$ is as follows:

$\Phi_{1}$ - the length $|\sigma|$ of $\sigma$ is a positive multiple of $K$, say $|\sigma|=(m+1) \cdot K$

$\Phi_{2}-\sigma$ can be written as $\sigma=\sigma_{0} \ldots \sigma_{m}$ with $\sigma_{0}, \ldots, \sigma_{m} \in \widehat{\Sigma}^{K}$ such that (a) is

satisfied, therein $\varphi(\ell \cdot K+1, p)$ holds precisely for $p=\ell \cdot K+s_{\ell}$

$\Phi_{3}$ - the first condition of (b) is satisfied

$\Phi_{4}-$ the second condition of (b) is satisfied

$\Phi_{5}-$ the third condition of (b) is satisfied 


\section{B.4 The Formula $E$ and the Interpretation $\left\langle f_{\amalg}, \mathcal{I}_{\amalg}\right\rangle$}

The formula $E\left(x_{1}, x_{2}\right)$ is defined as follows:

$$
\begin{aligned}
E\left(x_{1}, x_{2}\right) & =L\left(x_{1}, x_{2}\right) \wedge \exists X_{1} \exists X_{2}\left(\varphi_{\mathrm{Pre}}\left(x_{1}, X_{1}\right) \wedge \varphi_{\mathrm{Pre}}\left(x_{2}, X_{2}\right) \wedge \psi\left(X_{1}, X_{2}\right)\right) \\
\varphi_{\mathrm{Pre}}(x, X) & =\varphi_{\mathrm{Cl}}(x, X) \wedge \forall Y\left(\varphi_{\mathrm{Cl}}(x, Y) \rightarrow \forall y(y \in X \rightarrow y \in Y)\right) \\
\varphi_{\mathrm{Cl}}(x, X) & =x \in X \wedge \forall y \forall z(S(y, z) \wedge z \in X \rightarrow y \in X) \\
S(y, z) & =S_{0}(y, z) \vee S_{1}(y, z) \\
\psi\left(X_{1}, X_{2}\right) & =\forall z_{1} \forall z_{2}\left(z_{1} \in X_{1} \wedge z_{2} \in X_{2} \wedge L\left(z_{1}, z_{2}\right) \wedge\left(\exists y S\left(y, z_{1}\right)\right) \rightarrow \chi\left(z_{1}, z_{2}\right)\right) \\
\chi\left(z_{1}, z_{2}\right) & =\exists y_{1} \exists y_{2}\left(\left(S_{0}\left(y_{1}, z_{1}\right) \wedge S_{0}\left(y_{2}, z_{2}\right)\right) \vee\left(S_{1}\left(y_{1}, z_{1}\right) \wedge S_{1}\left(y_{2}, z_{2}\right)\right)\right)
\end{aligned}
$$

The ideas behind these formulae are the following:

$\varphi_{\mathrm{Cl}}(x, X)$ - the set $X$ contains $x$ and is closed under taking predecessors $\varphi_{\operatorname{Pre}}(x, X)-$ the set $X$ is the smallest one (w.r.t. inclusion) having the property $\varphi_{\mathrm{Cl}}(x, X)$ and hence $\amalg \bar{t} \models \varphi_{\operatorname{Pre}}((i, u), U)$ iff $U=\operatorname{Pre}(i, u)$

$\psi\left(X_{1}, X_{2}\right)$ - describes the condition on $\operatorname{Pre}(i, u)$ and $\operatorname{Pre}(j, v)$ in the characterisation of $|u|=|v|$ in terms of these two sets

Lemma B.1. For all $\bar{t} \in\left(T_{\Sigma}\right)^{n}$ there is an MSO-interpretation $\left\langle f_{\amalg}, \mathcal{I}_{\amalg}\right\rangle$ of $\otimes \bar{t}$ in $\amalg \bar{t}$ such that $f_{\amalg}(u)=(i, u)$, where $i$ is minimal with $u \in \operatorname{dom}\left(t_{i}\right)$, and $\mathcal{I}_{\amalg}$ does not depend on $\bar{t}$.

The formulae $\mathcal{I}_{\amalg}=\left(\Delta_{\mathcal{I}_{\amalg}} ;\left(S_{d}^{\mathcal{I}_{\amalg}}\right)_{d \in\{0,1\}},\left(P_{\bar{\alpha}}^{\mathcal{I}_{\amalg}}\right)_{\bar{\alpha} \in \widehat{\Sigma}_{\square}^{n}}\right)$ are as follows:

$$
\begin{aligned}
& \Delta_{\mathcal{I}_{\mathrm{II}}}(x)=\bigvee_{1 \leq i \leq n}\left(Q_{i}(x) \wedge \bigwedge_{1 \leq j<i} \varphi_{j, \square}(x)\right) \\
& S_{d}^{\mathcal{I}_{\mathrm{I}}}(x, y)=\Delta_{\mathcal{I}_{\mathrm{U}}}(x) \wedge \Delta_{\mathcal{I}_{\mathrm{U}}}(y) \wedge \exists z\left(S_{d}(z, y) \wedge E(x, z)\right) \\
& P_{\left(\alpha_{1}, \ldots, \alpha_{n}\right)}^{\mathcal{I}_{\amalg}}(x)=\Delta_{\mathcal{I}_{\mathrm{U}}}(x) \wedge \bigvee_{1 \leq i \leq n} \varphi_{i, \alpha_{i}}(x) \\
& \varphi_{i, a}(x)=\exists y\left(Q_{i}(y) \wedge P_{a}(y) \wedge E(x, y)\right) \quad(i \in[1, n], a \in \Sigma) \\
& \varphi_{i, \square}(x)=\neg \exists y\left(Q_{i}(y) \wedge E(x, y)\right) \\
& (i \in[1, n])
\end{aligned}
$$

\section{B.5 The Interpretations $\left\langle f_{\otimes, i}, \mathcal{I}_{\otimes, i}\right\rangle$}

Lemma B.2. For each $i \in[1, n]$ and all $\bar{w} \in\left(\widehat{\Sigma}^{\star}\right)^{n}$ there is an MSO-interpretation $\left\langle f_{\otimes, i}, \mathcal{I}_{\otimes, i}\right\rangle$ of $w_{i}$ in $H(\otimes \bar{w})$ such that $f_{\otimes, i}(p)=(p-1) \cdot n+i$ and $\mathcal{I}_{\otimes, i}$ does not depend on $\bar{w}$.

The formulae $\mathcal{I}_{\otimes, i}=\left(\Delta_{\mathcal{I}_{\otimes, i}} ; \leq^{\mathcal{I}_{\otimes, i}},\left(P_{\alpha}^{\mathcal{I}_{\otimes, i}}\right)_{\alpha \in \widehat{\Sigma}}\right)$ are as follows:

$$
\begin{aligned}
\Delta_{\mathcal{I}_{\otimes, i}}(x) & =x \equiv i(\bmod n) \wedge \neg \psi_{i, \square}(x) \\
\leq^{\mathcal{I}_{\otimes, i}}(x, y) & =\Delta_{\mathcal{I}_{\otimes, i}}(x) \wedge \Delta_{\mathcal{I}_{\otimes, i}}(y) \wedge x \leq y \\
P_{\alpha}^{\mathcal{I}_{\otimes, i}}(x) & =\Delta_{\mathcal{I}_{\otimes, i}}(x) \wedge \psi_{i, \alpha}(x) \\
\psi_{i, \alpha}(x) & =\bigvee_{\bar{\alpha}=\left(\alpha_{1}, \ldots, \alpha_{n}\right) \in \widehat{\Sigma}_{\square}^{n}, \alpha_{i}=\alpha} P_{\bar{\alpha}}(x) \quad\left(\alpha \in \widehat{\Sigma}_{\square}\right)
\end{aligned}
$$




\section{B.6 The Interpretation $\left\langle f_{H}, \mathcal{I}_{H}\right\rangle$}

Lemma B.3. For all $\bar{t} \in\left(T_{\Sigma, K}\right)^{n}$ there is an MSO-interpretation $\left\langle f_{H}, \mathcal{I}_{H}\right\rangle$ of $\amalg \bar{t}$ in $H(\otimes C(\bar{t}))$ such that $\mathcal{I}_{H}$ does not depend on $\bar{t}$.

The formulae $\mathcal{I}_{H}=\left(\Delta_{\mathcal{I}_{H}} ;\left(S_{d}^{\mathcal{I}_{H}}\right)_{d \in\{0,1\}},\left(P_{a}^{\mathcal{I}_{H}}\right)_{a \in \Sigma}, L^{\mathcal{I}_{H}},\left(Q_{i}^{\mathcal{I}_{H}}\right)_{1 \leq i \leq n}\right)$ are as follows:

$$
\begin{aligned}
\Delta_{\mathcal{I}_{H}}(x) & =\bigvee_{1 \leq i \leq n} \Delta_{\mathcal{I}_{\otimes, i}}(x) \\
S_{d}^{\mathcal{I}_{H}}(x, y) & =\bigvee_{1 \leq i \leq n}\left(\Delta_{\mathcal{I}_{\otimes, i}}(x) \wedge \Delta_{\mathcal{I}_{\otimes, i}}(y) \wedge\left(S_{d}^{\mathcal{I}_{C}}\right)^{\mathcal{I}_{\otimes, i}}(x, y)\right) \\
P_{a}^{\mathcal{I}_{H}}(x) & =\bigvee_{1 \leq i \leq n}\left(\Delta_{\mathcal{I}_{\otimes, i}}(x) \wedge\left(P_{a}^{\mathcal{I}_{C}}\right)^{\mathcal{I}_{\otimes, i}}(x)\right) \\
L^{\mathcal{I}_{H}}(x, y) & =\Delta_{\mathcal{I}_{H}}(x) \wedge \Delta_{\mathcal{I}_{H}}(y) \wedge \exists z(z \equiv 1(\bmod K \cdot n) \wedge z \leq x, y<z+K \cdot n) \\
Q_{i}^{\mathcal{I}_{H}}(x) & =\Delta_{\mathcal{I}_{\otimes, i}}(x)
\end{aligned}
$$

\section{B.7 The Sentence $\Gamma_{H}$}

The sentence $\Gamma_{H}$ is defined as follows:

$$
\begin{aligned}
\Gamma_{H} & =\Phi \wedge \bigwedge_{0 \leq i<n} \Psi_{i} \\
\Phi & =\forall x \forall y\left(x \equiv 1(\bmod n) \wedge x \leq y<x+n \rightarrow \bigvee_{\alpha \in \widehat{\Sigma}_{\square}^{n}}\left(P_{\alpha}(x) \wedge P_{\alpha}(y)\right)\right) \\
\Psi_{i} & =\forall x \forall y\left(x \leq y \wedge \psi_{i, \square}(x) \rightarrow \psi_{i, \square}(y)\right) \quad(i \in[1, n])
\end{aligned}
$$

where $\psi_{i, \square}$ is the formula from the construction of $\mathcal{I}_{\otimes, i}$ for $i \in[1, n]$. The formula $\Phi$ defines the set $H\left(\left(\widehat{\Sigma}_{\square}^{n}\right)^{\star}\right)$, whereas $\Psi_{i}$ defines the set of all words $w \in\left(\widehat{\Sigma}_{\square}^{n}\right)^{\star}$ with $\pi_{i}(w) \in \widehat{\Sigma}^{\star} \square^{\star}$, where $\pi_{i}:\left(\widehat{\Sigma}_{\square}^{n}\right)^{\star} \rightarrow\left(\widehat{\Sigma}_{\square}\right)^{\star}$ is the projection to the $i$-th component. Thus, the conjunction $\bigwedge_{i \in[1, n]} \Psi_{i}$ defines the language $\otimes\left(\widehat{\Sigma}^{\star}\right)^{n}$. Since $H\left(\otimes\left(\widehat{\Sigma}^{\star}\right)^{n}\right)=H\left(\left(\widehat{\Sigma}_{\square}^{n}\right)^{\star}\right) \cap \otimes\left(\widehat{\Sigma}^{\star}\right)^{n}, \Gamma_{H}$ defines this set. 


\section{Proof of Lemma 5.4}

Lemma 5.4 Let $\mathfrak{A}$ and $\mathfrak{A}_{1}, \ldots, \mathfrak{A}_{r}$ be infinite linear orderings with $\operatorname{dom}(\mathfrak{A})=$ $\operatorname{dom}\left(\mathfrak{A}_{1}\right) \times \cdots \times \operatorname{dom}\left(\mathfrak{A}_{r}\right)$ and satisfying the following two conditions:

(1) $\operatorname{cmp}_{\mathfrak{A}_{\mathfrak{A}}}(\bar{a}, \bar{b})$ is determined by $\operatorname{cmp}_{\mathfrak{A}_{1}}\left(a_{1}, b_{1}\right), \ldots, \operatorname{cmp}_{\mathfrak{A}_{r}}\left(a_{r}, b_{r}\right)$ for all $\bar{a}, \bar{b} \in A$,

(2) if $\bar{a}, \bar{b} \in A$ differ only in the $i$-th component, then $\operatorname{cmp}_{\mathfrak{A}}(\bar{a}, \bar{b})=\operatorname{cmp}_{\mathfrak{A}_{i}}\left(a_{i}, b_{i}\right)$.

Then, there exists a permutation $\pi$ of $\{1, \ldots, r\}$ such that $\mathfrak{A}$ is isomorphic to $\mathfrak{A}_{\pi(1)} \cdot \mathfrak{A}_{\pi(2)} \cdots \mathfrak{A}_{\pi(r)}$.

Proof. Let $\mathfrak{A}=(A ;<)$ and $\mathfrak{A}_{i}=\left(A_{i} ;<_{i}\right)$ for each $i \in[1, r]$. Consider $\bar{a}, \bar{b} \in A$ with $a_{i} \leq_{i} b_{i}$ for all $i \in[1, r]$. If we put $\bar{c}^{(k)}=\left(b_{1}, \ldots, b_{k}, a_{k+1}, \ldots, a_{r}\right)$ and apply condition (2) repeatedly, we obtain

$$
\bar{a}=\bar{c}^{(0)} \leq \bar{c}^{(1)} \leq \cdots \leq \bar{c}^{(r)}=\bar{b} .
$$

For the rest of this proof, fix $\bar{x}, \bar{y}, \bar{z} \in A$ such that $x_{i}<_{i} y_{i}<_{i} z_{i}$ for all $1 \leq i \leq r$ and put $\bar{e}^{(i)}=\bar{x}_{i / y_{i}}$. Since the $\bar{e}^{(i)}$ are mutually distinct, there is a permutation $\pi$ of $\{1, \ldots, r\}$ such that

$$
\bar{e}^{(\pi(1))}>\bar{e}^{(\pi(2))}>\cdots>\bar{e}^{(\pi(r))} .
$$

Due to condition (1), this permutation does not depend on the specific choice of $\bar{x}$ and $\bar{y}$ as long as $x_{i}<_{i} y_{i}$ for all $i \in[1, r]$. To see that this permutation has the desired property, we need show that the one-to-one correspondence

$$
f: \mathfrak{A} \rightarrow \mathfrak{A}_{\pi(1)} \cdots \mathfrak{A}_{\pi(r)},\left(a_{1}, \ldots, a_{r}\right) \mapsto\left(a_{\pi(1)}, \ldots, a_{\pi(r)}\right)
$$

is an isomorphism. However, to keep notation clear, we simply assume that $\pi$ is the identity.

Therefore, it suffices to show that $\bar{a}<\mathfrak{A}_{1} \cdots \mathfrak{A}_{r} \bar{b}$ implies $\bar{a}<\bar{b}$ for all $\bar{a}, \bar{b} \in A$. Thus, consider $\bar{a}, \bar{b} \in A$ satisfying the premise. Due to the definition of $\mathfrak{A}_{1} \cdots \mathfrak{A}_{r}$, there exists a $k \in[1, r]$ such that $a_{k}<_{k} b_{k}$ and $a_{i}=b_{i}$ for all $i \in[1, k-1]$. For all $i \in[1, r]$ we let $g_{i}=\min _{\mathfrak{A}_{i}}\left(a_{i}, b_{i}\right), h_{i}=\max _{\mathfrak{A}_{i}}\left(a_{i}, b_{i}\right)$

$$
\bar{c}^{(i)}=\left(g_{1}, \ldots, g_{i}, h_{i+1}, \ldots, h_{r}\right) \in A,
$$

and

$$
\bar{d}^{(i)}=\left(g_{1}, \ldots, g_{i-1}, h_{i}, g_{i+1}, \ldots, g_{r}\right) \in A .
$$

Using induction, we show $c^{(i)}<d^{(i)}$ for all $i \in[1, r]$ with $g_{i} \neq h_{i}$. Clearly, $g_{i} \neq h_{i}$ implies $g_{i}<_{i} h_{i}$. For the largest $i$ with this property, $c^{(i)}<d^{(i)}$ directly follows from condition (2). For all other $i$ with $g_{i} \neq h_{i}$ consider the least $j>i$ with $g_{j} \neq h_{j}$. We obtain the following chain of inequalities

$$
\bar{c}_{i / x_{i}, j / y_{j}}^{(i)}=\bar{c}_{i / x_{i}, j / y_{j}}^{(j)}<\bar{c}_{i / y_{i}, j / x_{j}}^{(j)}<\bar{d}_{i / y_{i}, j / y_{j}}^{(j)}<\bar{d}_{i / z_{i}, j / x_{j}}^{(i)},
$$


where the first and last inequality are due to $\bar{e}^{(j)}<\bar{e}^{(i)}$ and condition (1), whereas the second one is implied by the induction hypothesis $\bar{c}^{(j)}<\bar{d}^{(j)}$ and $(1)$. Finally, a last application of (1) yields $\bar{c}^{(i)}<\bar{d}^{(i)}$. Altogether, we obtain

$$
\bar{a} \leq \bar{c}^{(k)}<\bar{d}^{(k)} \leq \bar{b},
$$

where both non-strict inequalities use (|x|). 